\title{
A high order numerical method of MCM equation for image denoising
}

\author{
Guangyuan Chi ${ }^{1, \text { a }}$, Xiaozhong Yang ${ }^{2, b}$ \\ ${ }^{1}$ Mathematical and Physical Academy of North China Electric Power \\ University, Beijing, 102206, China \\ ${ }^{2}$ Mathematical and Physical Academy of North China Electric Power \\ University,Beijing, 102206, China \\ achiguangyuan1@126.com, byxiaozh@ncepu.edu.cn
}

\begin{abstract}
.
The nonlinear PDE model with special geometry, mean curvature motion (MCM) equation has been implemented by many methods in image processing. In this paper, the compact alternating direction implicit (CADI) method is constructed for MCM equation to denoise the noisy image, which is based on the alternating direction implicit (ADI) method. In theory, this paper is intended to discuss the properties of the CADI method in several aspects. The numerical experiments show that the effect of the CADI method for image denoising.

Keywords: image denoising; mean curvature motion (MCM) equation; $A D I$ method; compact alternating direction implicit (CADI) method; numerical experiment.
\end{abstract}

\section{Introduction}

Nowadays, the most widely used method of PDEs (Partial Differential Equations) in image processing [1], MCM equation, which shares the double features of diffusion and advection, is applied to various aspects of image denoising and smoothing [2].

The normal form of MCM equation is given as follows

$$
\left\{\begin{array}{c}
\frac{\partial u}{\partial t}=\kappa|\nabla u| \\
u(0, x, y)=u_{0}(x, y)
\end{array}\right. \text {. }
$$

Where $|\nabla u|=\sqrt{u_{x}^{2}+u_{y}^{2}}$ is the gradient modulus of each pixel in the image, and $\kappa=\left(u_{x x} u_{y}^{2}-2 u_{x} u_{y} u_{x y}+u_{y y} u_{x}^{2}\right) /\left(u_{x}^{2}+u_{y}^{2}\right)$ is the level set curvature of function $u$. 
Since MCM equation is a nonlinear diffusion equation, the analytic formula of the solution cannot be calculated in normal ways. Peter Smereka (2003) [3] proposed a good stability and accuracy method, the semi-implicit level set method, but the calculation of this method is very complex. ADI method [4] has been applied in image processing by many researchers [5]. For MCM equation, it is stable unconditionally and it has 2-order accuracy. On the basis of ADI method, this paper proposes the CADI method for image denoising contrary to MCM equation, with the 2-order accuracy in time and the 4-order accuracy in space, which improve the accuracy of numerical method. When the CADI method is used to process the noisy image, we can get a better result.

\section{Construction of CADI method Introduction}

The MCM equation can be written as

$$
\frac{\partial u}{\partial t}=\Delta u-N(u)
$$

It's easy to see that $N(u)=\left(u_{x x} u_{x}^{2}+2 u_{x} u_{y} u_{x y}+u_{y y} u_{y}^{2}\right) /\left(u_{x}^{2}+u_{y}^{2}\right)$ is a nonlinear second order operator. For $N(u)$, we know that $\quad N(u)=\nabla(|\nabla u|) \cdot \eta=(\partial|\nabla u|) /(\partial \eta) \quad$ and $\quad \eta=\nabla u /|\nabla u|$, where $N(u)$ is the gradient of the gradient magnitude of $u$,Therefore we know that, if $u$ is parameterized to be close to distance function, then the value of $N(u)$ is small [3]. During the process of solving the equation, a parameter $\beta$ is introduced to avoid the effect of the gradient $|\nabla u|=0$ on $N(u)$ such that the gradient is $|\nabla u|=\sqrt{u_{x}^{2}+u_{y}^{2}+\beta}$.

According to the reference [6], we construct the CADI method for MCM equation as follows

$$
\begin{aligned}
& \left(\mathcal{A}-\frac{k}{2} \delta_{x}^{2}\right) \bar{u}_{i, j}=\left(\mathcal{B}+\frac{k}{2} \delta_{y}^{2}\right) u_{i, j}^{n}+\frac{k}{2} \mathcal{B N}(u)_{i, j}^{n} . \\
& \left(\mathcal{B}-\frac{k}{2} \delta_{y}^{2}\right) u_{i, j}^{n+1}=\left(\mathcal{A}+\frac{k}{2} \delta_{x}^{2}\right) \bar{u}_{i, j}+\frac{k}{2} \mathcal{B} N(u)_{i, j}^{n} .
\end{aligned}
$$

Where $k$ is the time step, $\mathcal{A}$ and $\mathcal{B}$ are operators, $\bar{u}_{i, j}$ is the value of the transition layer, $\delta_{x}$ is the central difference operator, $\delta_{x} u_{i}=\left(u_{i+\frac{1}{2}}-u_{i-\frac{1}{2}}\right) / h$, and $h$ is the space step. In this paper, for each pixel $(i, j)$ in the image, we apply the central difference 


$$
\begin{aligned}
& \left(u_{x}\right)_{i, j}^{n}=\frac{u_{i+1, j}^{n}-u_{i-1, j}^{n}}{2 h},\left(u_{y}\right)_{i, j}^{n}=\frac{u_{i, j+1}^{n}-u_{i, j-1}^{n}}{2 h}, \\
& \left(u_{x x}\right)_{i, j}^{n}=\frac{u_{i+1, j}^{n}-2 u_{i, j}^{n}+u_{i-1, j}^{n}}{h^{2}},\left(u_{y y}\right)_{i, j}^{n}=\frac{u_{i, j+1}^{n}-2 u_{i, j}^{n}+u_{i, j-1}^{n}}{h^{2}}, \\
& \left(u_{x y}\right)_{i, j}^{n}=\frac{u_{i+1, j+1}^{n}-u_{i-1, j+1}^{n}-u_{i+1, j-1}^{n}+u_{i-1, j-1}^{n}}{4 h^{2}} .
\end{aligned}
$$

In order to solve the Eqs. (3)-(4), the image to be processed is treated as the initial condition (the first layer data). The intermediate layer data is obtained by Eq. (3), and then the next layer data directly by Eq. (4). Calculating in this way, a better denoised image will be got.

\section{The analysis of the CADI method}

\subsection{The existence of solutions analysis}

Eqs. (3)-(4) are symmetric about the variables $\mathrm{x}$ and $\mathrm{y}$. During the process of solving the Eq. (3), by setting $r=k / h^{2}$, obviously, we get the right-hand side vector $\mathrm{b}$ of equation $M x=b$ corresponding to Eq. (3), where the coefficient matrix $M$ is

$$
M=\left(\begin{array}{ccccc}
1+r & -\frac{r}{2} & & & \\
\frac{1}{12}-\frac{r}{2} & \frac{5}{6}+r & \frac{1}{12}-\frac{r}{2} & & \\
& \ddots & \ddots & \ddots & \\
& & \frac{1}{12}-\frac{r}{2} & \frac{5}{6}+r & \frac{1}{12}-\frac{r}{2} \\
& & & -\frac{r}{2} & 1+r
\end{array}\right) \in R^{(m+1) \times(m+1)} .
$$

We know that if a matrix is a strictly diagonally dominant matrix, then it is nonsingular. For any value of $r$, the tri-diagonal matrix $M$ has the unique solution. Therefore, Eq. (4) has the unique solution. For MCM equation in image denoising, we have the following theorem

Theorem 1: The solution of CADI method (3)-(4) for MCM equation (2) is existing and unique. 


\subsection{The convergence and stability analysis}

Lemma 1[6] : $f$ is the function about the variable $x$ and $y \cdot v=\left\{v_{i, j}^{k} \mid 0 \leq i, j \leq m\right\}$ is the solution of the CADI method (3)-(4). It gives $\left\|f^{n+\frac{1}{2}}\right\|^{2}=h^{2} \sum_{i, j=1}^{m-1}\left(f_{i, j}^{n+\frac{1}{2}}\right)^{2} \quad$, and then $\left|v^{n}\right|_{1}^{2} \leq \frac{3}{2}\left|v^{0}\right|_{1}^{2}+\frac{9}{4} k \sum_{l=0}^{n-1}\left\|f^{l+\frac{1}{2}}\right\|^{2}$.

During the process of image denoising, $N(u)$ can be calculated by explicit method, where the data bases on the precious layer of the image. Using Lemma 1 and the previous analysis, we obtain

Theorem 2: The CADI method (3)-(4) for MCM equation (2) is unconditionally stable and convergent, and the truncation error $O\left(k^{2}+h^{4}\right)$.

Hence, according to the theoretical analysis above, it can be enough to illustrate the superiority of our method.

\section{Application of the compact ADI method}

In the current field of image processing, there are a variety of objective evaluation criteria. In this paper, the peak signal to noise ratio (PSNR) is used to measure the quality of the denoised images. For an image of $m \times n$ in size, its PSNR can be computed by

$$
P S N R=10 \lg \left\{\frac{255^{2}}{\frac{1}{M N} \sum_{i=1}^{M} \sum_{j=1}^{N}[f(i, j)-\hat{f}(i, j)]^{2}}\right\}
$$

Where $f(i, j)$ is the original image and $\hat{f}(i, j)$ is the denoised image. All experiments are performed in Matlab on a laptop with an Intel Core Duo P7350 processor and $3.00 \mathrm{~GB}$ of memory. In numerical experiments, we choose the time step $k=0.2$ and space step $h=1$, and set parameter $\beta=0.02$.

\subsection{Numerical experiments}

For a 2-D gray image, the Salt and Pepper noise with the variance 0.08 is removed by ADI method and our method in Fig. 1, where the value of PSNR for the noisy image is $15.1693 \mathrm{db}$. 

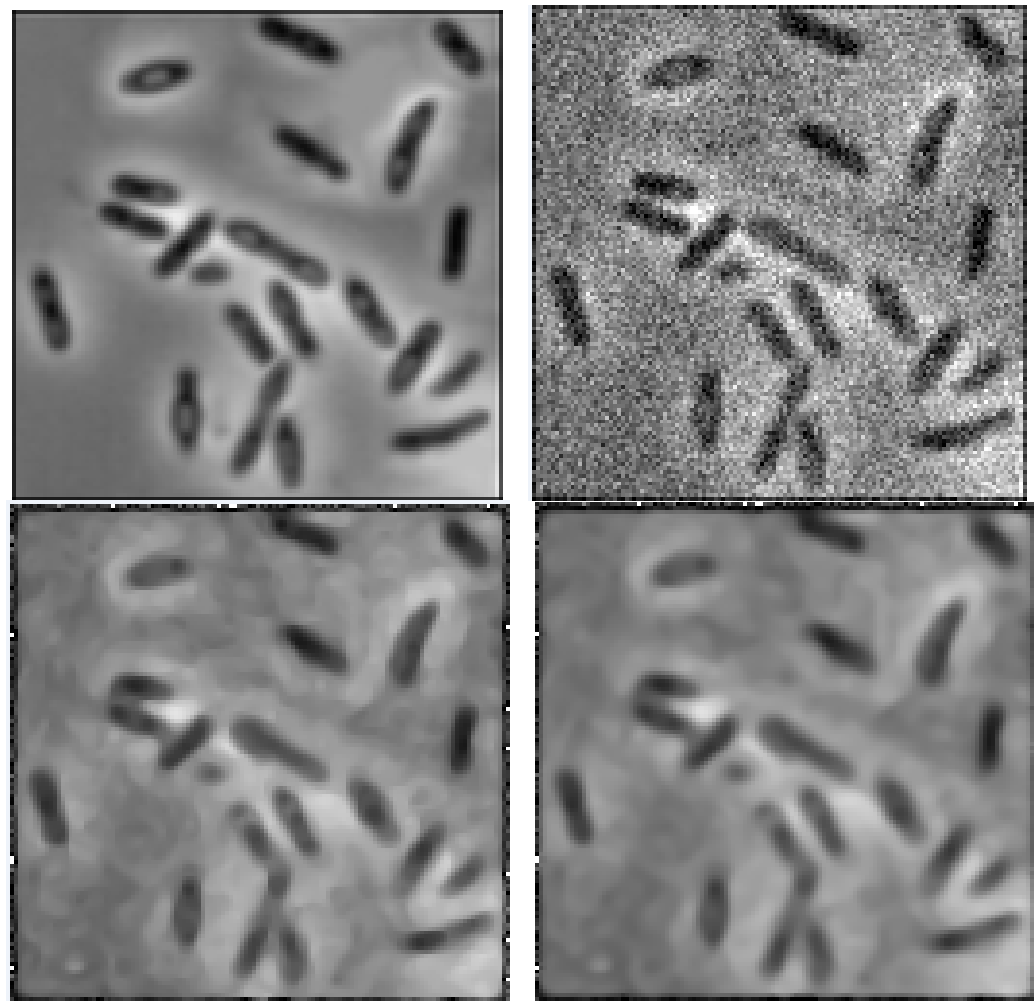

(a) original image

(b)noisy image

(c) result of ADI method

(d)result of CADI method

Fig. 1 The comparison of the output of two methods: (a) is the original image of the size $128 \times 128$. (b) is the noisy image corrupted by Salt and Pepper noise. (c) is the output of the ADI method with 15 iterations. (d) is the output of CADI method with 15 iterations.

In Fig. 1, we see that no obvious staircase effect appears during the processing of our method and ADI method. Both the two methods for MCM equation (2) in image denoising get better effect. In Tab. 1, by observing the evaluation criteria PSNR values, we find the values all fall within the interval 20-35db, which the quality of the denoised images is within the acceptable range of people [7]. Within the same conditions, by observing the images Fig. 1(c) and Fig. 1(d), we find the images denoised by our method is a little better than denoised by ADI method.

Tab. 1 The PSNR of the results

\begin{tabular}{|c|c|}
\hline Methods & The PSNR of Fig. $1[\mathrm{db}]$ \\
\hline ADI method & 22.3653 \\
\hline CADI method & 22.4133 \\
\hline
\end{tabular}




\subsection{Error checking}

In order to show that our method is better than ADI method in image denoising, we define a matrix. For an image of $m \times n$ in size, the matrix corresponding to the original image is $M$ and the matrix corresponding to the denoised image is $N$. To reflect the error between the original image and the denoised image, we set the matrix $L=\operatorname{abs}(M-N)$ and use the library function images in Matlab to get the figure corresponding to the matrix $L$.

In Fig. 2(a), the error matrix $L$ is obtained through Fig. 1(a) and Fig. 1(c). In Fig. 2(b), the error matrix $L$ is obtained through Fig. 1(a) and Fig. 1(d).
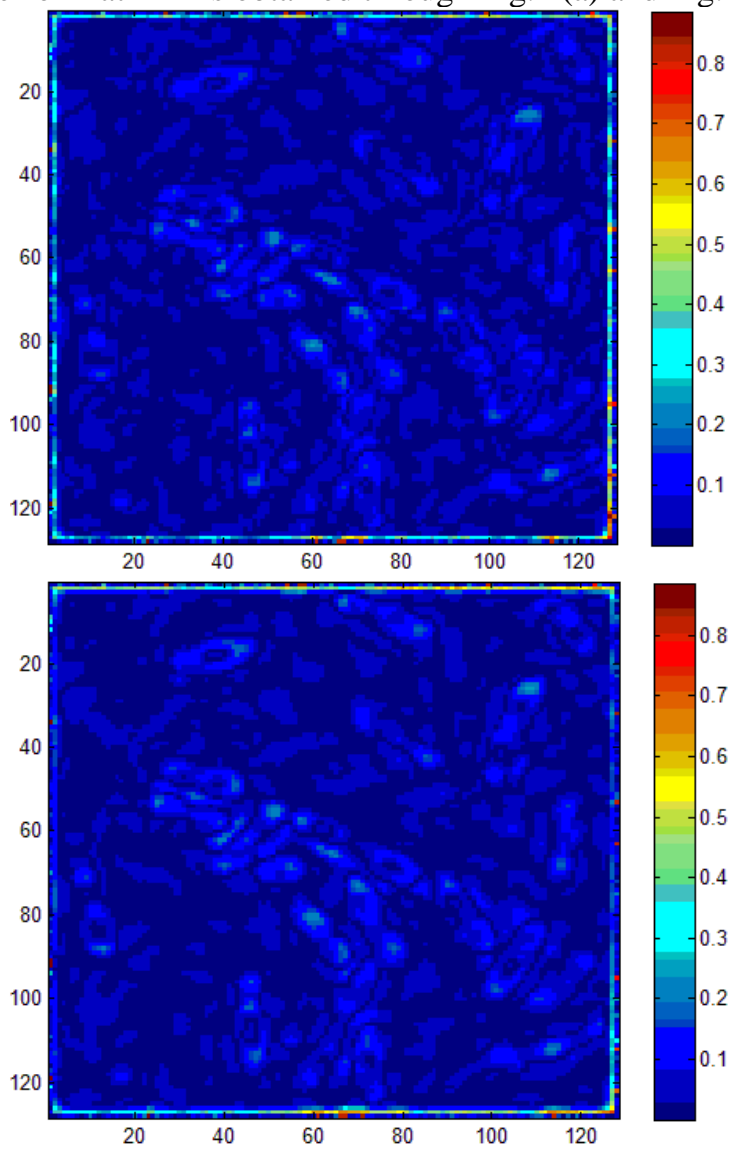

Fig. 2 (a)The error results of ADI method $\quad$ (b) The error results of our method

It can be visually seen from Fig. 2(a) and Fig. 2(b) that the error of the result by our method is less. In Fig. 1, the image denoised by our method can be better to approximate the original image. 


\section{Conclusions}

In this paper, a high order accuracy CADI method is proposed for MCM equation. The theoretical analysis shows that our method is stable unconditionally and its solution is existing and unique for the image denoising, in addition, the convergence of the method has 4-order accuracy in space. The error checking objectively illustrates that our method has higher accuracy. From Fig. 1, it gives that the images denoised by our method are obviously better than them by ADI method. By our method, it can be better to approximate the original image. The application of our method in this paper is promising and effective. Future works will focus on processing the nonlinear part and promoting of the CADI method on the field of image restoration.

\section{Acknowledgements}

This work was financially supported by the National Natural Science Foundation (11371135) and the Fundamental Research Funds for the Central Universities (2014ZZD10).

\section{References}

6. Chan T F, Shen J J. Image processing and analysis: variational, PDE, wavelet, and stochastic methods [M]. SIAM, 2005.

7. Du H W, Zhang Y. Numerical schemes of MCM equation for removing impulse noise [J]. Computer Engineering and Applications, 2010, 46(25), 164-167.(in Chinese)

8. Smereka P. Semi-implicit level set methods for curvature and surface diffusion motion [J]. Journal of Scientific Computing, 2003, 19(1): 439-456.

9. Witelski T P, Bowen M. ADI schemes for higher-order nonlinear diffusion equations [J]. Applied Numerical Mathematics, 2003, 45(2): 331-351.

10. Kim H, Calvert V R, Kim S. Preservation of fine structures in PDE-based image denoising [J]. Advances in Numerical Analysis, 2012.

11. Liao H L, Sun Z Z. Maximum norm error bounds of ADI and compact ADI methods for solving parabolic equations [J]. Numerical Methods for Partial Differential Equations, 2010, 26(1): 37-60.

12. A. Ichigaya, M. Kurozumi, et.al.. A method of estimating coding PSNR using quantized DCT Coefficients [J]. IEEE Transactions on Circuits and Systems for Video Technology, 2006, 16(2):251-259. 